\title{
Motivation and personal challenges while enrolled in higher education: The pathway to becoming a baccalaureate nurse
}

\author{
Sarah Kelly ${ }^{* 1}$, Kathleen Evanovick Zavotsky ${ }^{2}$, Erin Delany ${ }^{1}$, Claudia Pagani ${ }^{2}$, Christopher P. Duffy $^{2}$, Elisheba \\ Haqq-Stevens ${ }^{2}$, Myrna Young ${ }^{2}$ \\ ${ }^{1}$ School of Nursing, Rutgers, The State University of New Jersey, New Brunswick, United States \\ ${ }^{2}$ Robert Wood Johnson University Hospital, Robert Wood Johnson Barnabas Health System, New Brunswick, United States
}

Received: April 25, 2017

DOI: $10.5430 /$ jnep.v7n11p14
Accepted: May 30, 2017

Online Published: June 7, 2017

URL: https://doi.org/10.5430/jnep.v7n11p14

\begin{abstract}
Background: The exploration of the non-traditional or self-sufficient financially independent student experiences in higher education are considerably less researched, specifically addressing personal experience while pursuing higher education, when compared to traditional, those who recently graduated from high school, college students. Therefore, the objective of this study was to explore non-licensed hospital employees' (paraprofessional) perceptions related to their motivation to obtain a baccalaureate degree and overcome personal challenges to become a registered nurse.

Methods: A qualitative descriptive focus group research design was used to explore the perceptions of minority paraprofessional employees had related to their motivations and challenges to become a registered nurse. All student- paraprofessionals, who were funded through the Robert Wood Johnson grant program, were invited to participate in this study. A semi-structured interview guide was used to explore the participants' thoughts about obtaining a college degree and what their motivation is for obtaining a college degree in nursing. Informal questions were used to involve the participants in the discussion. The study was approved by the university's institutional review board and participants completed an informed consent prior to any data collection.

Results: Five participants were engaged in this study. The major theme that these participants reported was the different challenges and sacrifices (finances and life situations) that they had to deal with prior to starting courses and while they were currently taking courses. The second theme was motivation; these participants were motivated to have a better life.

Conclusions: These participants had to deal with certain challenges and make sacrifices in order to pursue their dream; however, the participants were able to find the needed motivation to achieve their dreams and become a registered nurse.
\end{abstract}

Key Words: Minority nursing students, Nursing education, Challenges and motivation

\section{INTRODUCTION AND BACKGROUND}

The exploration of higher education for the non-traditional student experience is considerably less researched than the traditional student experience, specifically addressing personal experience while pursuing higher education. According to the United States Department of Education's National
Center for Education Statistics, ${ }^{[1]}$ a non-traditional student is defined as having met one of the following seven features: delayed enrollment into postsecondary education; part-time college attendance, employed full time, is financially independent, has dependents regardless of a spouse, is a single parent, or does not have a high school diploma. Specific

*Correspondence: Sarah Kelly; Email: sekelly1972@gmail.com; Address: School of Nursing, Rutgers University, Newark, United States. 
topics of interest include target motivators to enter higher education, personal barriers, non-traditional modes of communication and student characteristics that lead to academic success despite personal challenges.

Many nontraditional students pursue higher education "for its own sake", wanting to increase their knowledge in hopes of being able to use that knowledge for the greater good. ${ }^{[2]}$ These students were interested in making a difference in people's lives, and less interested in pursuing an education as a means for a higher paying job. ${ }^{[2]}$ It is apparent that those returning to school may have different motivations from the start of the higher education process than traditional students, and could be important to consider these students face upcoming challenges and barriers.

Intrinsic motivation is defined as 'the doing of an activity for its inherent satisfaction rather than for some separate consequence," whereas extrinsic motivation focuses more on external reward. ${ }^{[3]}$ In one study looking at nontraditional students studying psychology, nursing, education, and "other", the students reported more intrinsic motivation towards their studies than extrinsic motivation, overall. The nursing students, however, reported significantly more extrinsic motivation than those studying psychology, namely for the benefit of career advancement. ${ }^{[3]}$ Looking further into intrinsic motivation, its link to positive affect, and determining what might foretell a student's affinity for both, Bye, Pushkar, and Conway ${ }^{[4]}$ discovered that interest is a strong predictor. This interest fuels lifelong learning due to the student's tendency to seek out knowledge. ${ }^{[4]}$

Another motivator that emerged through this study was the students' desire to set an example for their children. These students hope to create a better future for their children, and to also convey a positive attitude about obtaining a higher education. ${ }^{[2]}$ Although, the women particularly felt that pursuing higher education put a strain on their ability to fulfill household duties. Student mothers reported feeling that time spent doing school work was time spent away from family responsibility, especially for single parent families. ${ }^{[2]}$

There are other crucial factors that emerged as contributors to nontraditional students' motivation to return to school. Location and ease of commute were very important to this population, some claiming it to be one of the most important factors. ${ }^{[2]}$ Campus location had to be convenient and realistically fit into their routine in order for these students to believe that higher education is possible. ${ }^{[2]}$ Various studies indicate that commuters attempt to minimize their time spent commuting as much as possible, with an average commute time of about 30 minutes one way, and over $89 \%$ of students in one study reporting a weekly commute time to and from campus of 10 or less hours per week. ${ }^{[5]}$

The cost associated with both time and finances was a barrier reported by nontraditional students. Financial costs to the students encompassed tuition, travel, and course materials, lack of paid work, and childcare. Other costs related to time included time needed for other responsibilities, as mentioned above, and also time for socializing and self-care. ${ }^{[2]}$ Students reported associated feelings of guilt, anxiety and inadequacy related to being unable to uphold certain responsibilities to themselves and others. ${ }^{[2]}$ Biddix $^{[5]}$ affirms these challenges, declaring the ultimate consequence of these costs; "the reality of moving through for many commuter students is that, regardless of their desire and dedication to graduate, they may need to stop out as a result of family or financial priorities."

A few students in the study discussed how race and ethnicity were important in determining where they felt comfortable going to school. They reported that they did not want to go to a school that was all White, and that a good fit would have an ethnic mix. ${ }^{[2]}$ Research has found that students of color may prefer to commute to predominantly White college campuses, due to the continued social, cultural and spiritual support that their home communities provide. ${ }^{[5]}$ One factor that appears to contribute to nontraditional students' motivation in higher education was "a sense of community", ${ }^{2]}$ which is known to improve academic performance among students in higher education. $^{[5]}$

Evans $^{[6]}$ found that there was a strong cultural obligation to family that is common among these students. In addition, Young, Johnson, Arthur and Hawthrone ${ }^{[7]}$ suggested that students' culture influenced their ability to be successful in higher education. Young et al. ${ }^{[7]}$ looked at three different cultural groups, European Americans, African Americans and Hispanic Americans, exclusively, evaluating socio economic status, family generation with college experience and perceived social support as predictors for both intrinsic and extrinsic motivation for academics. They were able to identify that each cultural group is unique, stating that this finding "could be the basis to better understand and motivate individuals within each group to best encourage and inspire them. ${ }^{[7]}$ A common theme found by Evans ${ }^{[6]}$ in the barriers to educational success among minority students were the sacrifices that minority students had to make in order to meet their requirements for school and work. Minority students expressed that sacrificing time with family in order to meet the demands of school was a great concern for them. They also reported sacrificing study time in order to meet family obligations. ${ }^{[6]}$

After getting a glimpse at what might be some of the motivating factors for nontraditional students to enter, and subse- 
quently remain in higher education, it is relevant to note that there may also be underlying personal qualities and characteristics that are consistent with nontraditional students who are successful in pursuing a higher education. Duckworth and Gross $^{[8]}$ explore the concepts of self-control and grit, noting that these concepts, while different, are both associated with increased academic success. Between the two, grit alone has been shown to predict success more so than self-control. ${ }^{[8]}$ Grit differs from self-control in that someone demonstrating grit will tolerate setbacks and continue working towards the long-term goal, even if it takes years or decades. The time frame for both concepts is different. Self-control involves an immediate response to a stimulus, whereas grit refers to a persistent pursuit over a long period of time.

Duckworth and Gross ${ }^{[8]}$ argue that grit can be a better determinant of success than self-control. While grit and selfcontrol are often both exhibited in a successful person, when controlled for each concept, grit appeared to be a higher determinant of success. This finding can be interpreted to mean that those who have grit, but demonstrate less self-control, will tend to be more successful than those who exhibit selfcontrol without grit. Those who demonstrate grit also tend to delineate a subset of goals that work toward achieving the ultimate goal, and are able to pursue these goals with tenacity. ${ }^{[8]}$ Therefore, the purpose of this study was to explore non-licensed hospital employees' perceptions related to their motivation to obtain a baccalaureate degree and overcome personal challenges to become a registered nurse.

\section{METHOD}

\subsection{Design and sample}

A qualitative descriptive focus group research design was used to explore perceptions minority non-licensed hospital employees (paraprofessional) had related to their motivation to initiate and pursue a baccalaureate degree in nursing. The use of the focus group design provided the opportunity for these participants to openly elaborate on their perceptions and expand the discussion on their pursuit of a degree in nursing. ${ }^{9]}$ Eleven minority paraprofessionals, who were funded through the Unites States based, Robert Wood Johnson Foundation "New Paths to Professional Nursing" (NPPN) grant program, were invited to participate in this study.

The NPPN program provided financial resources, including reimbursement for classes, supplies, and text books, counseling services, and an academic tutor to qualified minority non-licenses hospital employees. This program was solely focused on providing support to students in order to complete their pre-requisites for entrance as junior year students into a baccalaureate nursing program. The tutor facilitated monthly meetings to prepare students to meet the challenges of academics. Topics discussed during those meetings, included balancing responsibilities and test taking strategies. In addition to the monthly peer meetings, the tutor facilitated individual meeting with each paraprofessional monthly. These individual meetings focused on developing a trust relationship and provided an opportunity to explore methods in overcoming personal barriers to success. The Institutional Review Board granted approval to conduct the study.

After informed consent was obtained, a member of the research team acted as the facilitator for the focus group. In addition, an observer was present to maintain field notes and manage the audio recording equipment. Each subject was issued a number and referred to that number throughout the focus group. The focus group meeting lasted approximately one to two hours and light refreshments were served during the meeting.

\subsection{Data collection}

A semi-structured interview guide was used to explore the participants' thoughts about obtaining a college degree and what their motivation is for obtaining a college degree in nursing. Informal questions were used to involve the participants in the discussion. Sample questions from the semi-structure interview guide included: What led you to decide that you needed to pursue further education? What are some of the challenges (at home and work) have you encountered as you pursue your educational goal? How has your attitude towards accomplishing your educational goal changed since you began the process?

The focus group was conducted in a private room at the university where students were currently enrolled in classes. The use of a private room decreased possible interruptions and allowed for good sound quality for the audio recording. Prior to the data collection, ground rules were established so all participants would have a chance to participate. The interviews lasted approximately 60-90 minutes and were audio recorded. Two members of the research team conducted the focus group, with one member taking field notes. The focus group interviews were conducted until repetitive information was obtained or data saturation occurred. The data were analyzed using content analysis.

\subsection{Data analysis}

After the focus group was complete, the audio recording was transcribed verbatim, into written text. The transcripts were checked against the audio recording for accuracy by the primary investigator (PI). Field notes were incorporated into the written narrative. Data were analyzed by four members of the research team, using Krueger and Casey's ${ }^{[10]}$ method including frequency, specificity, emotion, and extensiveness. 
After four members of the research team analyzed the transcriptions, the PI reviewed their findings. Multiple methods were used to establish data trustworthiness: peer debriefing and member checks (verification) were conducted after the analysis was completed. Each subject was assigned a number to maintain anonymity.

\section{Findings}

\subsection{Characteristics of participants}

Five female participants $(\mathrm{N}=5)$ were engaged in this study. From the data, two themes emerged: 1) challenges and sacrifices and 2) motivation. Each theme consisted of several codes. The codes for Challenges and sacrifices were: finances and life situations; whereas the codes for motivation were: better life, support from family and co-workers, and caring for others.

All of the participants were minority females, either African American or Hispanic, with an average age of 41, and had completed year one of the two year program. All of the subjects had completed some college credit in a university or community college setting. Several participants were single mothers and worked full-time at an urban academic medical center. Their positions within the medical center included clinical care technician, secretary and an outreach coordinator.

\subsection{Challenges and sacrifices}

The major theme that these participants reported was the different challenges and sacrifices that they had to deal with prior to starting courses and while they were currently taking courses.

\subsection{Finances}

All of the participants $(\mathrm{N}=5)$ identified the financial barriers they were constantly dealing with as a challenge to being able to go back to school and making ends meet while in school. Participant \#1

identified that it is very expensive place to live and I have to do everything by myself even though I have my parents supporting me. But it's really hard for them to help me economically, so I have to do everything by myself and to go to school now I have to cut a lot of things that you know I can't afford.

\section{Participant \#5 stated}

when I tried and started school I had to stop because of money and then I started again and then I had to stop again because of money. So I had always let the need for money, because, I have to survive, and I have to feed my family and I am the main provider that is what would stop me from being able to continue and finally be successful.

The participant then proceeded to report that she "has a second job because... with two kids in college, [she] cannot live on the [her current] job [salary].” Participant \#3 supported the idea that her financial situation was the primary reason she was "putting it [enrolling in classes] off for the past three years, just because of the financial part and where to get the funds." This statement was further echoed by participant \#2, "you know, financially there was a little bit of a hindrance there, you know. The money I was making was going towards my living expenses and there wasn't a whole lot left over, you know to pursue education." She further reported that "I was a single parent... as a parent, your priority. . you know is your children..."

However, even after participation in this grant, which supplemented the healthcare organizations tuition reimbursement program with funding for classes, participants had trouble meeting their financial obligations. Participant \#5 elaborated by saying

it was difficult on most weeks... I had my lights shut off this past winter. I was grateful, I went to family members' house to do my work to have Wi-Fi, my children ended up staying at their friend's house in order to do homework and not be in the dark.

In addition, participant \#3 stated that

Before being part of this grant I was working 96 hours biweekly and then having to drop to 48 [to attend classes], I had to cut a lot of my meetings. I tried to make ends meet so it was like I really have to keep a tight budget, a tight schedule of what I can do, what I cannot do. Also what another person stated about the financial strain where it comes out of our pocket [they had to pay the tuition first and get reimbursed], that I was a big challenge for me.

Participant \#2 and \#4 concurred with the above sentiment. Participant \#4 reported that "for the 2 nd summer class I had to ask for a letter from the grant saying that the money would be paid and that allowed me to go ahead and do the class..." Finances were a common concern for all participants; however, it was not the only challenge they were dealing with. 


\subsection{Life situations}

Another issue facing a few of these participants was the desire to attend school, but the inability to do so because of life circumstances. Participant \#5 shared that "once I knew that I was going to be a mom I still wanted to continue my education, but once baby \#2 came along, then that was a no go for me." She had to prioritize and at the time, as young, new mother, she had to make sacrifices and school was not the priority at the time. Another example was participant \#4's desire to go to school, yet unable to obtain that goal due to her life situation. Having been raised on a British Island, “... when I came here [US]... that [nursing school] was the first thing I was headed for... so then husband, kids, life, all of that put it [nursing school] on the back burner." The desire to go to school was an important component to these participants'ability to be successful.

Although the desire to go to school was important, it's only part of the process, being able to effectively manage their work/life/school balance was a continuing challenge for many.

Participant \#4 noted that

I finished work at 11 at night, I am dead tire[d], I cannot study or [do] anything because my job entails a lot of walking I am walking maybe 7 hours out of the 8 hours that I am here [at work]. Its only when I sit to actually make [time to study] I am sitting for dinner or whatever. And again I am tired when I go home.

She was not the only person who suggested that studying and getting sleep was a concern. Participant \#5 acknowledged that "... I would lose sleep because [I] had to study... I was very anxious and had a lot of anxiety...so you have to adapt you know..."

These participants realized what their end goal was and in order to achieve that goal, sacrifices had to be made. For example, participant \#5 stated

so, [my] social life had to go, family life had to go except for my immediate family [daughter, son, and dog]... mom and dad got a phone call every now and then, or you know my extended family... I come from a collective culture and with that being said its no one calls to tell you that they are coming over. People just show up, and that is my culture. So, that had to stop unfortunately... I did not go into any family gatherings, [I did] nothing.

Another participant recognized that she needed to remove 18 herself from her current situation in order to be successful in school. So, participant \#4 put her plan into action

I decided to do a lifestyle change. I decided to move from where I was. I am [cutting] down to just living in a room and so I can have more finances at my disposal and whenever the grant kicks in it kicks in. But I [now] have extra money to pay my school fees upfront... and [I] just keep my head down stay focused, more focused... I don't have to worry about. . . other distractions, or little kids, no boyfriends, no nothing...

These participants had many challenges to overcome and sacrifices to make in order to pursue their goal to become a nurse. Their ability to successfully navigate through these experiences, demonstrates the motivation they had to achieve their dream.

\subsection{Motivation}

The second overarching theme was motivation; who or what motivated the students to obtain a higher educational degree. These participants were motivated to have a better life. The influence to have a better life came from family, co-workers, or the desire to help others overcome a health problem.

\subsection{Better life}

Several participants were motivated by the fact that they wanted a better life and being a nurse could facilitate a fulfilling, rewarding career. Participant \#5 stated that "as a nurse... you have opportunities, the connections. I mean nursing is so well founded, so well founded like is has such an amazing ... solidarity to it. It's almost like you want to conquer the work and save everybody." Participate \#3 wants a better life- "better future, better me, just a better future overall, a better version of me." One participant described that it is "time for me". She put her dreams on hold to care for her family and it's her time now. She wants to" go back to school to further [her] education. One stated that she was going back to school at this time in her life because "it could be about her, and she wanted to accomplish this goal before she was eligible for retirement". Although this fulfilled her own desire, another motivating factor was, that when she was engaged in higher education, it may have an influence on her own children and grandchildren, so that "they can get you know good jobs". Wanting to accomplish personal goals and promoting a positive image for others can motivate a person to continue when the experiences get hard. 


\subsection{Support from family and co-workers}

Another motivating factor for the participants was the family support to start school and continue taking classes, even when they were overwhelmed by the circumstances. Four of the participants identified that in different ways their family motivated them to reach for their goal of becoming a nurse. Participant \#3 wanted to provide a better future for her family. She suggested that watching her mother work as a nurse to provide a better lifestyle, encouraged her "to give my kids [a better future] as well. So family has always been my motivation." The support one has from their family is also a motivating factor to continue. A participant acknowledged that without her family, she would not be able to do this. She described that "when I am always down they always say you can do it... my mom always says, you know, even if I say I can't, she is like you can push for it, do better.. " Participant \#1 agreed that her mother motivated her to continue in school. She identified that

[my mom] went through a lot in her life and she is, even though, she went through cancer, she finished her school... she has always been my motivation... and sometimes I call her all frustrated, I say I cannot do this... she said, you know you can do it, if I did it you can. She has always been there for me to support me and help me.

One of the participant's children is a registered nurse and according to the participant, encouraged her to go back to school. According to the participant her daughter stated "mom you can do this, you got me through this, you can definitely do this for yourself". Participant \#1 believes that both her family and co-workers motivate her to continue in school. She informed the focus group that she tried to quit school a few times, but her support system (family and co-workers) said "no you can do it, and they motivated me to continue to this nursing program." Family support and encouragement have helped these participants to stay on their educational journey, but they are not the only source of support, as co-workers and peers have also played an integral part in motivating them along their educational pathway.

Many participants acknowledged that support came from coworkers. Participate \#1 acknowledged that her co-workers "gave me [her] a push to do it [apply for the grant]" and are also a huge source of support;

I am not doing this alone, that if I really need help, I can call someone and they will get back to me, and will help me wherever I am student. So having that motivation was a huge boost... it boosted my confidence and told me that yeah you can do this...

Participant \#3 similarly stated that "the nurses [were] also pushing me, and they told me to just do it. What do you have to lose?" Participate \#1 uses her co-workers as her motivation, "I have been using them to motivate myself to continue to become a nurse." One participant had an exchange with the assistant vice-president (VP) of the healthcare organization and according to the participant, the assistant VP stated "come on... I believe in you, I believe in you, you can do this, you can do this..." These participants look for motivation from their family and co-workers.

\subsection{Caring for others}

These participants identified the ability to improve the health of others was a motivating factor for pursuing the nursing field. Participant \#1, \#2, and \#4 described the emotional connection they have for wanting to care for those in-need and improve their health. According to participant \#2, “... nursing. . . if you don't have a heart for other people, then it's probably not a profession that you should consider." She expanded and stated "I get a lot of enjoyment out of being able to be there to help people through some of the most fearful and most vulnerable times of their lives." Interestingly, Participant \#4 suggested that "you want to be there, talking them through, helping them be strong for the family members". Witnessing the changes patients experience during their health crisis and the integral part that nurses play during a health recovery is an influential experience for those who want to help others.

\subsection{Discussion}

This study explored five paraprofessional employees' perceptions of their reentry into the educational system and their ability to obtain a baccalaureate degree in nursing. These participants are focused on achieving their lifelong goal-becoming a nurse. During this study two themes emerged that influenced the participants' ability to be successful. They had to deal with certain challenges and make sacrifices in order to pursue their dream; however, the participants were able to find the needed motivation to achieve their dreams.

The first theme, challenges and sacrifices focused on the different circumstances that have influenced participants' ability to enroll in higher education and maintain a balance among their school and work schedule and their family life obligations. This idea supported findings from Vaccaro and Lovell's ${ }^{[11]}$ study. Adult students who desire to return to school are under a unique challenge to balance the demands 
of family and work with school. ${ }^{[11]}$ The demand that women experience as they attempt to fulfill their goals can put an undue stress on them-thus, influencing their ability to be successful.

Burrow, Mairs, Pusey, Bradshaw, and Keady ${ }^{[12]}$ identified that a consistent challenge among adults who decided to obtain a higher educational degree, was the ability to balance work, school, and family effectively. Adult students, who have a family, work, and attend school, have to walk a fine line so that they don't alienate any part of their life. These participants were in a similar position and had to carefully navigate and juggle expectations and experiences.

One of the main benefits for these participants, which differ from other studies - was the use of an academic tutor. The tutor met with each participant during the year to provide encouragement, provide time management advice, and study habits. Specifically, the tutor's role provided an outlet for the participants to share their concerns and issues-individually or in a group setting.

Another challenging aspect for many of these participants was their financial situation. These participants were able to receive financial assistance to enroll in courses and while attending classes; however, all of the participants were from a lower socioeconomic status and many were the primary care providers for their family. It is not uncommon for students to have financial concerns while going back to school. Financial stressors have affected many from fulfilling their goals. In a recent Australian study exploring stressors among 1,000 undergraduate students, financial hardships was one of four stressors identified. ${ }^{[13]}$

In a study of 363 American undergraduate students, Young et al. ${ }^{[7]}$ suggested that a student's socioeconomic status and cultural background had a combined effect on their success and motivation. The researchers reported that culture, specifically, African American and Hispanic cultures, had an impact on enrolling in college, staying in college, and graduating from college. Many of our participants had prior college credit, but for various reasons, were unable to complete their degree on time. In addition to the differences in college completion among the different cultures, Young et al. ${ }^{[7]}$ found that minority students with a lower socioeconomic status affected their ability to be successful in school. In the NPPN program the students were all minorities, were of a lower socioeconomic status, and required financial assistance in order to enroll in classes.

Although there were many challenges and sacrifices that these participants had to deal with, they were able to find the motivation that influenced their ability to achieve their goals.
These participants wanted a better life but also realized that they needed a push to achieve that life. Improving one's economic situation and thereby improving their life situation, is a common thread found in the literature. ${ }^{[14,15]}$

Merrill's ${ }^{[14]}$ study tells the story of two adult learners from the United Kingdom who choose to return to school in order to obtain a higher educational degree. Both women shared their stories of why they left school but also their reasons for returning to school. As seen in our current study, these two women had personal challenges and expectations that influenced their education; however, given the opportunity, each were determined to obtain that degree. They wanted a better life and economic stability. Swain and Hammond ${ }^{[15]}$ identified 13 different reasons why students are motivated during college. The 18 adults in their study described many motivating factors that coincided with each other. However, an overriding theme among seven of the motivating factors was geared towards improving their life-identified as extrinsic motivations. ${ }^{[15]}$ Although our study consisted of 5 participants, the findings support the idea that an important motivating factor to go back to school and obtain a higher degree, is the desire to improve one's life-through a better career.

There are economical, personal, and social motivations that influence students desire to go back to school. ${ }^{[15]}$ All of those motivations apply to our participants. For many in our study, obtaining a Bachelor of Science (BS) degree was a personal goal-a fulfillment of a dream. However, it also promoted a positive image for others; they became role models for their children and grandchildren.

This study supported the notion that motivation comes from various sources. Our participants found the motivation form their families and co-workers. Burrows et al. ${ }^{[12]}$ provided evidence indicating that support can come from families and co-workers. The ability to successfully meet their goals and the needs of others, puts adult learners in a challenging position-something that these participants had to deal with on a daily basis. As Williams and Seary ${ }^{[16]}$ found, resiliency is the key to adult learners being successful and reaching their goals.

\section{CONCLUSION AND NURSING IMPLICA- TIONS}

Minority students have added challenges in completing baccalaureate programs due to language barriers and increased cultural and family demands. The overarching themes identified in the study were congruent with what is in the literature. Students enrolled in the NPPN program had various supportive components, including a dedicated academic tutor, the 
nursing education department, medical librarian, and guaranteed employment. This level of support provided facilitated academic success for these participants. The removal of the stress and fear was important for these participants so that they could focus on career development and advancement. The hospital's commitment for career development was projected as genuine intention to improve paraprofessionals'life situation. Programs like this have a strong potential to improve employee engagement.

The Institute of Medicine [IOM] report ${ }^{[17]}$ on The Future of Nursing: Leading Change, Advancing Health, provides a map to transform the nursing profession to enhance quality to meet the needs of diverse populations. The report addresses the need to diversify the health care workforce. According to Reinhart and Hassmiller ${ }^{[18]}$ approximately $33 \%$ of the United States (US) population is part of a racial or ethnic minority group, yet only $18 \%$ of our nursing students are minorities. By 2050, African Americans, Asians, Latinos, and American Indians/Alaskan Natives will comprise a majority of ourpopulation. We need to make sure the nursing profession reflects the patients it serves, and that all nurses deliver culturally competent care.

There are many benefits in creating a program to assist paraprofessional employees to enter the nursing profession. The benefits are not just for the student but medical center and academia as well. Benefits for the medical center include growing employees from within the company, provide opportunities for career advancement, hire nursing staff that meets the cultural needs of the patients, improve patient sat- isfaction scores, improve employee engagement scores and lastly create a pipeline of nurses for the future. Academia benefits by enrolling engaged, motivated students who want to help others. Lastly the student benefits through career development, advancement, and the ability to fulfill lifelong dreams.

\section{Limitations}

There were several limitations to this study. The use of participants currently involved with the pathways to professional nursing program that enabled financial assistance for college classes could have biased the finding of this study. In addition, the use of a focus group could have influenced participants' ability to provide true perceptions of their experiences. Some of the participants may have been influenced by others statements and less likely to share their true perceptions. The small sample size and the use of a qualitative method also limits the ability to generalize the results to other populations. Cultural differences between the researchers and students could have also influenced their responses to certain questions; however, this was accounted for by having multiple members participate in the data collection with a similar cultural background.

\section{ACKNOWLEDgements}

This stufy was funded by the Robert Wood Johnson Foundation: Path to Professional Nursing Grant ID: 71976.

\section{CONFLicts OF INTEREST Disclosure}

The authors declare that there is no conflict of interest.

\section{REFERENCES}

[1] The Condition of Education. Washington, DC: U.S. Department of Education, National Center for Education Statistics; 2002.

[2] Reay D, Ball S, David M. 'It's Taking Me a Long Time but I'll Get There in the End': Mature students on access courses and higher education choice. Br Educ Res J. 2002; 28(1): 5-19. https : //doi.org/10.1080/01411920120109711

[3] Shillingford S, Karlin NJ. The role of intrinsic motivation in the academic pursuits of nontraditional students. New Horiz Adult Educ Hum Dev. 2013; 25(1): 91-102. https ://doi.org/10.1002/nh a3. 20033

[4] Bye D, Pashkar D, Conway M. Motivation, interest, and positive affect in traditional and nontraditional undergraduate students. Adult Educ Q. 2007; 57(2): 141-158. https://doi.org/10.1177/07 41713606294235

[5] Biddix JP. Understanding and Addressing Commuter Student Needs: New Directions for Student Services, Number 150. Somerset, New Jersey: Jossey-Bass; 2015.

[6] Evans BC. Attached at the umbilicus: Barriers to educational success for Hispanic/Latino and American Indian nursing students. J Prof
Nurs. 2008; 24(4): 205-217. PMid:18662656 https://doi.org/ $10.1016 / j$.profnurs .2007 .06 .026

[7] Young A, Johnson G, Arthur H, et al. Cultural and socioeconomic differences in academic motivation: A self-deterministic approach. JBER. 2011; 9: 37-46.

[8] Duckworth A, Gross JJ. Self-control and grit: Related but separate determinants of success. Curr Dir Psychol Sci. 2014; 23(5): 319-325. PMid:26855479 https://doi.org/10.1177/09637214145414 62

[9] Crabtree BF, Miller WL. Doing qualitative research 2nd edition. Thousand Oaks, CA: Sage Publications; 2011.

[10] Krueger RA, Casey MA. Focus Groups: A Practical Guide for Applied Research 3rd edition. Thousand Oaks, CA: Sage Publications; 2000. https://doi.org/10.1037/10518-189

[11] Vaccaro A, Lovell C. Inspiration from home: Understanding family as key to adult women's self-investment. Adult Educ Q. 2010; 60: 161-176. https://doi.org/10.1177/0741713609336111

[12] Burrows S, Mairs H, Pusey H, et al. Continuing professional education: Motivations and experiences of health and social care professional's part-time study in higher education. A qualitative liter- 
ature review. Int J Nurs Stud. 2016; 63: 139-145. PMid:27615415

https://doi.org/10.1016/j.ijnurstu.2016.08.011

[13] Karimshah A, Wyder M, Henman P, et al. Overcoming adversity among low SES: A study of strategies for retention. Aust Univ Rev. 2013; 55(2): 5-14.

[14] Merrill B. Determined to stay or determined to leave? A tale of learner identities, biographies and adult students in higher education. Stud High Educ. 2014; 40(10): 1859-1871. https : //doi .org/10 $.1080 / 03075079.2014 .914918$

[15] Swain J, Hammond C. The motivations and outcomes of studying for part-time mature students in higher education. Int J Lifelong Educ.
2011; 30(5): 591-612. https://doi.org/10.1080/02601370.2 011.579736

[16] Williams J, Seary K. I feel like I'm being hit from all directions: Enduring the bombardment as a mature-age learner returning to formal university. Aust J Adult Learn. 2011; 51(1): 119-142.

[17] Institute of Medicine. 2010. The future of nursing: Leading change, advancing health. Available from: http://books.nap.edu/open book.php?record_id=12956\&page $=$ R1

[18] Reinhard S, Hassmiller S. The future of nursing: Transforming health care. AARP The Journal. Available from: http://journal . aarpinternational .org/a/b/2012/02/ The-Future-of-Nursing-Transforming-Health-Care 